Castro Figueroa, A., Farfán Buitrago, D., y Olis Barreto, I., (2013). Vida y muerte empresarial. Estudios empíricos de perdurabilidad: el Grupo Nutresa. Revista Lebret (5). Bucaramanga,Colombia: Universidad Santo Tomás, pp 317 - 329.

\title{
Vida y muerte empresarial. Estudios empíricos de perdurabilidad: el grupo Nutresa*
}

\section{Life and Death of Business. Empirical Studies of Sustainability: The Nutresa Group}

\author{
Andrés Mauricio Castro Figueroa \\ Dalsy Yolima Farfán Buitrago ${ }^{2}$ \\ Irma María Olis Barreto
}

\begin{abstract}
Resumen
Basado en el comportamiento de las empresas como organismos dinámicos, el biólogo y epistemólogo chileno Humberto Maturana (2010) hace una analogía entre la teoría de la Biología Cultural, la cual dice que los organismos vivos se van modificando porque necesitan adaptarse para poder seguir viviendo. Lo mismo sucede con las empresas, las cuales deben ir cambiando según los requerimientos de los nuevos tiempos para poder sobrevivir. Esta investigación tomó como marco de referencia teorías desarrolladas sobre la perdurabilidad empresarial que se han asociado con el término "supervivencia" y con el pensamiento de autores como Peters y Waterman (1982), quienes fueron los primeros que publicaron un texto relacionado con el éxito empresarial. En el artículo se muestran los resultados del estudio para las empresas que conforman el grupo empresarial Nutresa.
\end{abstract}

\section{Palabras clave}

Perdurabilidad, Alimentos, Sostenible, Caso empresarial, Competencia

\section{Códigos de Clasificación JEL: M13}

\begin{abstract}
Based on the behavior of firms as dynamic organisms, chilean biologist and epistemologist Humberto Maturana (2010) makes an analogy between the theory of Cultural Biology, which says that living organisms are modified, because they need to adapt in order to keep on living. The same goes for companies, which must be changed according to the requirements of the times to survive. This research took as a framework developed theories on corporate sustainability, which have been associated with the term "survival" and thr thought of authors such as Peters and Waterman (1982) who were the first who published a text related to business success. The article results of the study for the companies that make the Nutresa business group.
\end{abstract}

Keywords

Sustainability, Food, Sustainable, Business Case, Competition

* Este estudio se enmarca dentro de la investigación con el mismo título adelantada por el Grupo de Investigación en Perdurabilidad Empresarial (GIPE) de la Universidad del Rosario, Colombia.

1 Magíster en Gestión de Organizaciones de la Universidad de Quebec. Profesor Principal de Carrera de la Universidad del Rosario, Colombia. Correo electrónico: andres.castro@urosario.edu.co

2 Magíster en Derecho Económico de la Pontificia Universidad Javeriana, Colombia. Profesora Auxiliar de Carrera de la Universidad del Rosario. Colombia. Correo electrónico: dalsy.fafan@urosario.edu.co

3 Magíster en Desarrollo Educativo y Social, Universidad Pedagógica Nacional. Coordinadora Proyección Social Facultad de Administración, Universidad del Rosario, Colombia. Correo electrónico: irma.olis@urosario.edu.co 
Castro Figueroa, A., Farfán Buitrago, D., y Olis Barreto, I., (2013). Vida y muerte empresarial. Estudios empíricos de perdurabilidad: el Grupo Nutresa. Revista Lebret (5). Bucaramanga,Colombia: Universidad Santo Tomás, pp 317 - 329.

\title{
Vida y muerte empresarial. Estudios empíricos de perdurabilidad: el grupo Nutresa*
}

\section{Life and Death of Business. Empirical Studies of Sustainability: The Nutresa Group}

\author{
Andrés Mauricio Castro Figueroa \\ Dalsy Yolima Farfán Buitrago ${ }^{2}$ \\ Irma María Olis Barreto
}

\begin{abstract}
Resumen
Basado en el comportamiento de las empresas como organismos dinámicos, el biólogo y epistemólogo chileno Humberto Maturana (2010) hace una analogía entre la teoría de la Biología Cultural, la cual dice que los organismos vivos se van modificando porque necesitan adaptarse para poder seguir viviendo. Lo mismo sucede con las empresas, las cuales deben ir cambiando según los requerimientos de los nuevos tiempos para poder sobrevivir. Esta investigación tomó como marco de referencia teorías desarrolladas sobre la perdurabilidad empresarial que se han asociado con el término "supervivencia" y con el pensamiento de autores como Peters y Waterman (1982), quienes fueron los primeros que publicaron un texto relacionado con el éxito empresarial. En el artículo se muestran los resultados del estudio para las empresas que conforman el grupo empresarial Nutresa.
\end{abstract}

\section{Palabras clave}

Perdurabilidad, Alimentos, Sostenible, Caso empresarial, Competencia

\section{Códigos de Clasificación JEL: M13}

\begin{abstract}
Based on the behavior of firms as dynamic organisms, chilean biologist and epistemologist Humberto Maturana (2010) makes an analogy between the theory of Cultural Biology, which says that living organisms are modified, because they need to adapt in order to keep on living. The same goes for companies, which must be changed according to the requirements of the times to survive. This research took as a framework developed theories on corporate sustainability, which have been associated with the term "survival" and thr thought of authors such as Peters and Waterman (1982) who were the first who published a text related to business success. The article results of the study for the companies that make the Nutresa business group.
\end{abstract}

Keywords

Sustainability, Food, Sustainable, Business Case, Competition

* Este estudio se enmarca dentro de la investigación con el mismo título adelantada por el Grupo de Investigación en Perdurabilidad Empresarial (GIPE) de la Universidad del Rosario, Colombia.

1 Magíster en Gestión de Organizaciones de la Universidad de Quebec. Profesor Principal de Carrera de la Universidad del Rosario, Colombia. Correo electrónico: andres.castro@urosario.edu.co

2 Magíster en Derecho Económico de la Pontificia Universidad Javeriana, Colombia. Profesora Auxiliar de Carrera de la Universidad del Rosario. Colombia. Correo electrónico: dalsy.fafan@urosario.edu.co

3 Magíster en Desarrollo Educativo y Social, Universidad Pedagógica Nacional. Coordinadora Proyección Social Facultad de Administración, Universidad del Rosario, Colombia. Correo electrónico: irma.olis@urosario.edu.co 


\section{Introducción}

Este estudio es resultado de un proceso de investigación desarrollado a partir del proyecto "Vida y muerte empresarial. Estudios empíricos de perdurabilidad" implementado por el Grupo de Investigación en Perdurabilidad Empresarial (GIPE), inscrito en la Línea de Investigación de Realidad Empresarial con el programa Epidemiologia Empresarial de la Escuela de Administración de la Universidad del Rosario. La Escuela se concentró en el reconocimiento a los esfuerzos empresariales de aquellos colombianos que con su ejemplo y resultados han consolidado el crecimiento de sus organizaciones enmarcados en factores de rentabilidad financiera, responsabilidad social y sostenibilidad. Dicha sostenibilidad, usualmente resulta en la creación de riqueza para la nación, en empleo para la población colombiana y de ejemplo para las nuevas generaciones de empresarios. En este contexto, se trataba de comprender el patrón y modelo que ha permitido perdurar a las empresas que ganaron o han estado postuladas al "Premio Empresario Colombiano del Año Mariposa de Lorenz". Este premio ejemplifica la forma como las organizaciones perduran en el tiempo a pesar de las fuerzas externas y su contundencia; cómo se desarrollan, crecen y evolucionan en tiempos de bonanza y en tiempos de dificultad. Reconoce el fortalecimiento de los mecanismos que los empresarios implementan para fomentar una cultura hacia la perdurabilidad empresarial que conduzca a una mayor productividad y competitividad en los sectores económicos del país.

Para ello, se realizó un análisis comparativo entre el modelo de selección del Premio y los trece componentes de medición de condiciones de perdurabilidad y, como resultado, se abordó el esquema y guía mental de la empresa de estudio que le ha dado reconocimiento y permanencia en los mercados. Se incluyó, además, un estudio de dinámica de sistemas para identificar el comportamiento de las empresas ganadoras del premio y el análisis del comportamiento de la empresa a partir de los resultados financieros, con base en los componentes de perdurabilidad. Como resultado, se pretende identificar el patrón y modelo mental que les ha permitido a las empresas permanecer en los mercados. El artículo se enfoca fundamentalmente en los resultados obtenidos para las empresas del Grupo NUTRESA.

\section{Fundamentos teóricos}

Dentro del tema de la problemática empresarial es necesario abordar la búsqueda e identificación de ¿cuáles son los principios administrativos que distinguen a las compañías perdurables de las que no lo son? Este interrogante surge de la observación de los sectores, en los cuales existen ganadores y perdedores, pero ambos se encuentran en un entorno con las mismas características. 
El término de perdurabilidad ha sido abordado desde diferentes autores, entre estos Ramírez, Muñoz y Pulido (1998, p. 10), quienes consideran importante "valorar y comprender una característica fundamental de las organizaciones, su capacidad para permanecer en entornos de diferente complejidad y con grados de turbulencia bastante disímiles". Estos autores buscan una definición que reúna las condiciones que tienen las empresas para perdurar en el tiempo, para identificar características que resultan esenciales para el sostenimiento y éxito de las mismas, y que permita ver cómo a través de los cambios enfrentan problemas que hacen que su gestión sea cada vez más compleja.

El caso de las empresas colombianas ilustra aspectos relacionados con la perdurabilidad y su administración. El Departamento de Planeación Nacional en Colombia (DNP) señala que la pequeña y la mediana empresa difícilmente sobreviven sus primeros años. Según datos recientes, el $41 \%$ no alcanzan al primer año y el $76 \%$ el quinto. La perdurabilidad de las empresas familiares no es mejor. El $35 \%$ sobrevive a la primera generación y el $13 \%$ a la tercera. La búsqueda de respuestas a este hecho conduce a una hipótesis preliminar: las empresas colombianas en su administración, no sólo adolecen de una gestión eficiente de su productividad, sino que además es evidente la ausencia del componente estratégico en el pensamiento de sus dirigentes (Restrepo, 2009).

En el libro Empresas Triunfadoras o en su nombre original The Winning Corporation: Management Practices That Work (Jagoe, 1987), se plantea que la "muerte" de muchas empresas es innecesaria y se puede evitar y, asimismo, presenta una serie de pautas para ayudar a los administradores a planificar el bienestar empresarial en todos los niveles. A su vez, Collins y Porras (1997) publican los hallazgos de una investigación en su libro denominado "Built to Last", seleccionando compañías "visionarias" en lugar de simplemente prósperas o duraderas, para reflejar el hecho de que se han distinguido como un tipo muy especial y selecto de instituciones. Son efectivamente más que prósperas y duraderas, son lo "mejor de lo mejor" en sus respectivas industrias y lo han sido desde hace decenios. Las condiciones establecidas para realizar la investigación fueron: Instituciones líderes en su industria, muy admiradas por personas conocedoras de negocios, que hayan dejado huella indeleble en el mundo en que vivimos, haber tenido múltiples generaciones de presidentes, haber pasado por múltiples ciclos de vida de productos y ser fundadas antes de 1950.

Una década después, Collins (2001) publica "Empresas que Sobresalen" en su nombre en inglés Good to great, en el cuál plasma los resultados de la investigación que buscó identificar las características de las organizaciones que lograron convertir la mediocridad en superioridad. 


\section{Metodología}

El propósito del estudio fue identificar el papel del empresario como guía y estratega en la organización, para lograr no sólo la sostenibilidad de la empresa en el tiempo, sino su éxito en los mercados.

Para efectuarlo, se seleccionó el sector de alimentos - dinámico y de gran impacto socioeconómico en Colombia - tomando como referencia el caso del Grupo Nacional de Chocolates - hoy conocido como GRUPO NUTRESA, el cual ha demostrado durante varias décadas su liderazgo y calidad de vida dentro del sector de alimentos y la perdurabilidad, tanto a nivel nacional como internacional, haciéndolo acreedor de una reputación y ejemplo empresarial para la industria de alimentos. Esta es una de las razones para realizar un estudio acerca de los diferentes factores que hacen que esta compañía se caracterice por la gestión y perdurabilidad en el tiempo. Como apoyo a esta investigación, se realizó una entrevista semiestructurada al Presidente de la organización, junto a una encuesta estructurada a los empleados de cargo profesionales y directivos, en total cuarenta y dos en Bogotá, para así poder analizar las condiciones de perdurabilidad en su interior. Con la entrevista realizada al Presidente del Grupo Nutresa, se vislumbró un panorama amplio sobre las actividades de este grupo y cómo ha logrado ser una de las empresas más exitosas y perdurables del país, que además, ha sido galardonada con el Premio Empresario Colombiano del Año "Mariposa de Lorenz" 2009, entregado por Escuela de Administración de la Universidad del Rosario.

\section{Análisis de datos}

La perdurabilidad es vista como un componente esencial en el desarrollo de las empresas, por esto la Escuela de Administración de la Universidad del Rosario se ha interesado por abordar este tema a fondo, planteando un estudio de "Condiciones para perdurabilidad", que le permitirá entender desde diferentes escenarios el comportamiento de las empresas a través del tiempo. En el marco de las empresas que han estado postuladas al "Premio Empresario del Año Mariposa de Lorenz", se identificaron dos ejes fundamentales que se deben observar: la Dirección y la Gerencia ${ }^{5}$, con el fin de identificar y comprender el patrón y modelo que le ha permitido ser sostenibles en el tiempo. El planteamiento de la Dirección cobra relevancia debido a que los empresarios que se encuentran al frente de su organización deben prospectar el futuro, orientar su consecución y observar la información que

4 Proyecto de investigación, Universidad del Rosario, Escuela de Administración, Grupo de Investigación en Perdurabilidad, realizado a 318 empresas de Colombia, que pertenecen a los sectores agroindustrial, alimentos, automotor, confecciones, editorial, farmacéutico, financiero, plásticos, servicios, telecomunicaciones y textiles. (2005).

5 Información tomada de la página web www.urosario.edu.co 
el entorno le presenta para lograr convertirla en estrategias coherentes y alcanzar un posicionamiento. Entendida así la temática, los trece factores de la perdurabilidad establecidos por la Escuela de Administración se describen en la Tabla 1.

En relación a la Dirección del Grupo Nutresa, en los últimos años se han tomado decisiones que les ha permitido enfrentarse al mundo globalizado, y han sido alineadas de acuerdo a la filosofía de especialización por áreas y objetivos estratégicos de internacionalización para el grupo empresarial, lo que les ha permitido diversificación del riesgo, posicionamiento de marca y distribución en el mercado objetivo. Con respecto a la Gerencia, buscan expandir su mercado, por esto incursiona en el mercado de valores de Colombia como un mecanismo para mejorar su plan de financiamiento y crecimiento. También se enfocan en procesos como: permanente mejora en las relaciones que tiene el grupo con compañías externas como proveedores y clientes, y en procesos internos para mejorar la calidad de los productos, infraestructura, contando con más de doscientos mil puntos de venta donde se distribuyen los productos de las diferentes unidades de negocios. Atienden también con prioridad la gestión humana, siendo muy selectivos a la hora de contratar un nuevo empleado, destacando las cualidades de emprendedores y líderes, seguros de sus opiniones y que puedan aportar a las decisiones de la empresa.

\section{Análisis del sector alimentos en Colombia}

El sector Agroindustrial es importante para el país ${ }^{6}$, debido a que le aporta al PIB nacional el 29\% de la producción total, generando a su vez el $21 \%$ del empleo en Colombia. El sector de alimentos en Colombia se compone de manera porcentual de la siguiente forma: el 29\% corresponde a productos de molinería, el $20 \%$ a productos lácteos, el $18 \%$ a carnes y pescados, el $15 \%$ a aceites, hortalizas, flores y grasas, el $8 \%$ a productos de panadería y el 10\% representa a otros subsectores. En 2010, el sector de alimentos creció a una tasa anual del 2,3\% y el de bebidas se contrajo a una tasa del 2,5\%. Ambos sectores crecieron en este período por debajo del promedio de la industria que creció a una tasa del 4,5\%. Durante el primer semestre de 2011, el crecimiento del sector de alimentos fue de 3,1\%, levemente inferior al promedio industrial que fue del 3,5\% especialmente por los sectores de molinería y almidones, refinación de azúcar, y transformación y conservación de carne y pescado.

El crecimiento del sector de alimentos desde el 2011 ha sido impulsado por la reactivación de la demanda interna, y por el dinamismo de las exportaciones que han sido muy dinámicas desde ese período. El crecimiento del sector ha sido frenado en los últimos años por las emergencias invernales que afectan especialmente a sectores como el de productos cárnicos, refinación de azúcar, productos lácteos y bebidas. Al ubicar el sector de molinería, panadería y almidones, se puede afirmar que ha

6 Ver Sector Agroindustrial Colombiano. http://www.inviertaencolombia.com.co 
presentado un crecimiento sobrio debido al costo de la materia prima proveniente del exterior, entre las que se encuentran el trigo, principal insumo para la producción, el maíz y la soya. Pero dentro de este sector sobresale de manera favorable el subsector de galletería, debido al importante volumen de exportaciones. Asimismo, se encuentra el subsector de cacao y chocolatería que ha presentado durante los últimos años una actividad creciente, en gran medida debido a su innovación, comercialización, diversificación y diferenciación de productos en el territorio nacional y su expansión a otros países. Aunque este sector ha sido negativamente afectado por la volatilidad y aumento en los precios del azúcar y la leche, los precios poco competitivos del cacao y las limitaciones en materia de producción, así como la implementación de mejores tecnologías, ha logrado sostenerse dentro de los más importantes y que aportan de manera significativa al PIB del país.

\section{Historia del Grupo Nutresa}

Desde el punto de vista histórico, este grupo antiqueño fue fundado en 1916 por varios empresarios inicialmente como productora de galletas y confetis bajo el nombre de "Fábrica Nacional de Galletas y Confites". En 1933 se hizo accionista por medio de la adquisición de nueva tecnología y fabricación de la marca Dux de Galletas Noel. En 1958, como parte de su estrategia de Grupo se crearon las Marcas Sello Rojo y Colcafé, con el fin de comercializar café tostado, soluble y molido, generando así más ingresos a la compañía. En 1960 se fusionaron dos filiales dándole como origen a la Industria Colombia de Café S.A, ese mismo año adquirieron Alimentos Zenú y Alimentos Cárnicos. En 1968 realizaron una adquisición nacional con la compra de la Fábrica Café de la Bastilla, la cual se transformó en Tropical Coffee Company. En 1970 aumentaron las adquisiciones a nivel nacional, identificando la primera en Barranquilla donde compró Frigorífico Continental y la segunda en Bogotá a través de Frigorífico Suizo con el fin de mejorar la oferta de productos cárnicos. En 1980 adquirió y fundó dos empresas, de galletas y cárnicos, Molinos de Santa Marta como proveedor de harina de trigo y Tecniagro. Con la apertura económica de los años 90, comenzaron a incursionar en el mercado internacional fundando comercializadoras en Venezuela y Ecuador. En 1997 adquirieron los Productos Alimenticios Doria, empresa líder en el mercado de las pastas; en 1999 realizó un Joint Venture vendiéndole a Danone el 30\% de Galletas Noel con el fin de generar un aliado estratégico.

A principios del nuevo milenio se dio paso a la venta de los productos por medio de “canales alternativos", caracterizados por máquinas dispensadoras y por catálogos. El año 2002 fundó una nueva comercializadora en México, la cual le abría paso para llegar a este país así como varias escisiones con el fin de mejorar la participación en los mercados, y en el 2004 las comercializadoras Cordialsa en Estados Unidos, Panamá, 
Guatemala, Costa Rica, Nicaragua, Honduras y El Salvador. Se compraron las fábricas de galletas y chocolates de Nestlé en Costa Rica, posicionando a la Compañía Grupo Nacional de Chocolates y Grupo Nacional de Galletas en este mercado. En 2005 la casa matriz se hizo dueña del $100 \%$ de las filiales adquiriendo de nuevo el $30 \%$ de Galletas Noel, y se fundaron dos nuevas filiales, como es el caso de Pastas Commarico y Setas de Colombia; y a nivel internacional se adquirieron Galletas Pozuelo en Costa Rica y Blue Ribbon en Panamá. En 2007 con ansias de mejorar la eficiencia y efectividad comercial, se fusionaron las redes de distribución de Nicaragua y Panamá. En 2008 se generó un Joint Venture con De La Recetta y Alpina para darle mayor cubrimiento al mercado colombiano. En 2009 se creó el Grupo Nutresa de México, y en el 2010 adquirió galletas dulces llamada Ferh Holdings LLC. Finalmente, en el 2011 se adquirió en República Dominicana la compañía especializada en helados llamada Helados Bon. En este mismo año, el Grupo Nacional de Chocolates dejó de llamarse así para cambiar a Grupo Nutresa, debido a que su propósito era una imagen y razón social que reflejara todas sus unidades de negocio y el compromiso que tiene con la nutrición.

\section{Resultados}

¿Por qué el Grupo Nutresa es una empresa perdurable? De acuerdo al contexto de la organización, el Grupo Nutresa es una empresa con objetivos empresariales orientados a la competitividad y por ende, a la perdurabilidad. Los objetivos estratégicos están enfocados a seguir creciendo exitosamente, fortaleciendo factores como competitividad, salud financiera, cultura empresarial, capacidad de innovación, capacidad de transformación y de mirar al futuro. La adquisición de talento humano joven y renovado; las marcas de la empresa que han cambiado de acuerdo a la época y necesidades del consumidor; la existencia de un solo código de Gobierno corporativo continuo donde la empresa se constituye como la única sociedad inversionista y se incrementa la eficiencia y la simplicidad; la capacidad de la compañía de transformarse y adaptarse a los tiempos, el que Grupo Nutresa sea una compañía con una capacidad de distribución que abarca el territorio nacional colombiano y todos los países donde se ha expandido y por último, la innovación de sus productos, procesos y modelo de negocios que la diferencian en el mercado, son factores definitivos para que clasifique dentro de las empresas perdurables.

Como resultado, el Grupo Nutresa expone sus teorías frente a los 13 ítems de perdurabilidad: 


\begin{tabular}{|c|c|}
\hline $\begin{array}{c}1 . \\
\text { Identidad de la } \\
\text { organización: }\end{array}$ & $\begin{array}{l}\text { La identidad organizacional en particular son los valores, comportamientos éticos y la } \\
\text { responsabilidad social identificados al interior de la organización. Cabe resaltar que } \\
\text { los valores que componen la filosofía y la actuación corporativa son: la autonomía y } \\
\text { coherencia estratégica, el buen gobierno corporativo, la competitividad, el desarrollo } \\
\text { de los trabajadores, el desarrollo sostenible, la ética, la gestión de la innovación y } \\
\text { el conocimiento, la participación, agilidad y gestión colaborativa, el respeto y la } \\
\text { seguridad alimentaria. Con estos componentes de cultura organizacional se encuentran } \\
\text { comprometidos los } 28.500 \text { trabajadores del Grupo Nutresa, lo que hace que la } \\
\text { organización esté orientada a un mismo propósito, con políticas de gestión alineada } \\
\text { con los resultados extraordinarios y a la adaptabilidad frente al cambio del entorno del } \\
\text { mercado, factores claves para llegar al camino de la perdurabilidad. }\end{array}$ \\
\hline $\begin{array}{c}2 . \\
\text { Formalización } \\
\text { para el gobierno }\end{array}$ & $\begin{array}{l}\text { El Grupo Nutresa se ha caracterizado por una longevidad de } 90 \text { años; para las empresas } \\
\text { líderes del mundo el promedio es de } 40 \text { años, por lo cual este indicador refleja la buena } \\
\text { salud empresarial de la compañía. Durante los } 90 \text { años de vida de la compañía, el } \\
\text { gobierno corporativo ha sido continuo y no generacional. Al ser continuo, significa que } \\
\text { los principios de la identidad organizacional han evolucionado con el tiempo de acuerdo } \\
\text { a la demanda del mercado y a los marcos regulatorios. }\end{array}$ \\
\hline $\begin{array}{c}3 . \\
\text { Cohesión social } \\
\text { para la acción }\end{array}$ & $\begin{array}{l}\text { La cohesión social se vivencia en la organización a través de los incentivos que se otorgan } \\
\text { al alcanzar resultados. Dentro de estos incentivos se encuentran las bonificaciones, que } \\
\text { dependen efectivamente del éxito en ventas. La medición del clima organizacional } \\
\text { muestra que la organización se encuentra en un nivel sobresaliente. Esto comprueba } \\
\text { que la fuerza de la cultura organizacional a través de una evaluación sobre la claridad } \\
\text { en los objetivos, las condiciones de trabajo, el apoyo del jefe directo, la remuneración, el } \\
\text { reconocimiento, la formación y la capacitación así como talento motivado y formado, se } \\
\text { encamina a obtener los estándares más altos. }\end{array}$ \\
\hline $\begin{array}{l}4 . \\
\text { Formalización: } \\
\text { soporte para las } \\
\text { decisiones: }\end{array}$ & $\begin{array}{l}\text { A lo largo de su vida organizacional, el grupo se ha caracterizado por la especialización } \\
\text { en diferentes áreas que le han permitido responder rápidamente a cambios en el entorno } \\
\text { bajo un enfoque de gobierno corporativo continuo. Bajo la creación en el } 2006 \text { de } \\
\text { Servicios Nacional de Chocolates S.A, el centro de servicios compartidos del Grupo y } \\
\text { con la fusión en el } 2007 \text { de las redes de distribución Cordialsa y Pozuelo en Nicaragua } \\
\text { y Panamá respectivamente, ha logrado mayor eficiencia y efectividad comercial para la } \\
\text { organización. Por otro lado, los lineamientos establecidos para la toma de decisiones } \\
\text { del grupo, han propiciado la supervivencia de la compañía en Venezuela, gracias } \\
\text { a la ideología políticamente neutral y el mantenimiento de precios inferiores a los de } \\
\text { la competencia, lo que ha mitigado los posibles riesgos de expropiación por parte del } \\
\text { gobierno de este país. }\end{array}$ \\
\hline $\begin{array}{c}5 . \\
\text { Reconocimiento } \\
\text { por el entorno y el } \\
\text { Sector: }\end{array}$ & $\begin{array}{l}\text { Este ítem de perdurabilidad se encuentra íntimamente relacionado con el objetivo } \\
\text { estratégico de crecimiento y liderazgo en el mercado por parte del grupo; objetivo } \\
\text { orientado a incrementar el tamaño y la participación del mismo. Para lograr este objetivo } \\
\text { se requiere ser líderes, hoy el Grupo Nutresa se constituye como un seguidor de esta } \\
\text { filosofía, sumado al objetivo estratégico de internacionalización, el cual está orientado } \\
\text { a la diversificación y a la disminución de la concentración en un solo mercado, con el } \\
\text { fin de reducir vulnerabilidades de la empresa y, por ende, al incremento del tamaño del } \\
\text { mercado objetivo. }\end{array}$ \\
\hline $\begin{array}{c}6 . \\
\text { Diferenciación }\end{array}$ & $\begin{array}{l}\text { En los últimos años se han realizado catorce adquisiciones de compañías nacionales } \\
\text { e internacionales por parte del grupo, lo que refleja innovación, nuevas marcas y un } \\
\text { portafolio de productos mucho más fuerte y sólido en un mercado cada vez más incierto. } \\
\text { Se destaca que esta diferenciación se fundamenta en la creación de valor agregado y } \\
\text { no en el sostenimiento de bajos costos, lo que garantiza que el Grupo Nutresa no se vea } \\
\text { afectada por los efectos de la revaluación y por los altos costos que significa la existencia } \\
\text { de la protección efectiva negativa para el sector agrícola y, por tanto, posea un margen } \\
\text { de rentabilidad para soportar los fenómenos externos y sobrevivir. }\end{array}$ \\
\hline
\end{tabular}




\begin{tabular}{|c|c|}
\hline $\begin{array}{l}\text { D. } \\
\text { Dinámica social de } \\
\text { los empleados }\end{array}$ & $\begin{array}{l}\text { Uno de los objetivos estratégicos del Grupo Nutresa está orientado al desarrollo de una } \\
\text { filosofía de gestión humana enfocada en la vinculación de la persona y el camino del } \\
\text { conocimiento dentro del factor humano, con el propósito de atraer y retener el talento } \\
\text { dentro de la organización. } \\
\text { La empresa propicia condiciones para la eficiencia y calidad en la acción de sus empleados } \\
\text { mediante la participación activa y el empoderamiento que les da en las decisiones, así } \\
\text { como la integración de grupos de trabajo, la consolidación de la cooperación y del } \\
\text { trabajo en equipo. De esta manera, resulta una dinámica social producto de la interacción } \\
\text { social y la autonomía. } \\
\text { El empoderamiento en los empleados que comparten un proceso de trabajo cualquiera, } \\
\text { hace que estos asuman la responsabilidad por la marcha de la actividad, tomando todas } \\
\text { las medidas que resulten necesarias, siempre que estén alineadas con la misión de su } \\
\text { unidad, la que a su vez debe ser congruente con la misión general de la empresa. } \\
\text { Al generar las condiciones propicias para la retención de talento humano, es claro que } \\
\text { las raíces de la cultura organizacional van a estar más arraigadas en una organización } \\
\text { que propicie el bienestar de sus empleados, lo que conlleva a trabajar con mayor } \\
\text { perseverancia por los objetivos comunes y la misión y visión de la organización. }\end{array}$ \\
\hline $\begin{array}{l}8 . \\
\text { Factores que } \\
\text { aportan a la } \\
\text { eficiencia }\end{array}$ & $\begin{array}{l}\text { El paso del gobierno corporativo de sociedades anónimas a holdings, donde el Grupo } \\
\text { Nacional Chocolates es el dueño único del } 100 \% \text { de las filiales, permitió la reducción } \\
\text { de juntas directivas innecesarias, creando juntas nominales y reducción de los auditores } \\
\text { externos, dejando un sólo inversionista llamado GNC (Grupo Nacional de Chocolates). }\end{array}$ \\
\hline $\begin{array}{c}9 . \\
\text { Consolidación }\end{array}$ & $\begin{array}{l}\text { La consolidación del grupo es resultado de la construcción de una organización con una } \\
\text { visión de futuro que hacen sus directivos, así como del compromiso y participación de } \\
\text { los empleados en ella. Es así como el objetivo para el } 2015 \text { es proporcionar calidad de } \\
\text { vida al consumidor con productos que satisfagan sus aspiraciones de bienestar, nutrición } \\
\text { y placer, lo que refleja crecimiento, consolidación y perdurabilidad. }\end{array}$ \\
\hline $\begin{array}{c}10 . \\
\text { Gestión Integral }\end{array}$ & $\begin{array}{l}\text { Dentro de los objetivos estratégicos del Grupo Nutresa se encuentra el desarrollo } \\
\text { sostenible que comprende responsabilidad social y ambiental. En este sentido, el grupo en } \\
\text { el } 2006 \text { creó la Fundación Grupo Nacional de Chocolates, con el propósito de concentrar } \\
\text { los esfuerzos de las compañías del Grupo en su contribución al desarrollo económico y } \\
\text { social de la comunidad. Adicionalmente creó el Centro de Investigación en Nutrición, } \\
\text { Salud y Bienestar Vidarium, que posteriormente se transforma en la Corporación que } \\
\text { lleva su mismo nombre en el } 2008 \text {. }\end{array}$ \\
\hline $\begin{array}{l}11 . \\
\text { Conocimiento } \\
\text { del entorno y el } \\
\text { mercado }\end{array}$ & $\begin{array}{l}\text { Bajo cada línea de negocio, el Grupo Nutresa cuenta con equipos e investigación y } \\
\text { desarrollo que continuamente intentan mejorar los productos y los ajustan a las nuevas } \\
\text { tendencias del mercado. }\end{array}$ \\
\hline $\begin{array}{l}12 . \\
\text { Eficiencia en } \\
\text { procesos }\end{array}$ & $\begin{array}{l}\text { Los métodos de gestión se materializan con la restructuración del gobierno corporativo, } \\
\text { en donde día a día, con el crecimiento y adquisiciones que el Grupo Nutresa realiza } \\
\text { a lo largo de Latinoamérica, se genera un enfoque especializado donde prima el } \\
\text { empoderamiento de los empleados en todos los niveles, llevando a que exista eficiencia } \\
\text { al interior de la organización. }\end{array}$ \\
\hline $\begin{array}{l}13 . \\
\text { Gestión } \\
\text { Financiera }\end{array}$ & $\begin{array}{l}\text { Uno de los objetivos estratégicos de la organización es el crecimiento rentable. Este } \\
\text { objetivo permite que el consumidor adquiera los productos, buscando no elevar tanto } \\
\text { el nivel de apalancamiento conservador e inferior al } 2 \% \text { para el grupo. Esto debido a } \\
\text { que para la organización un alto nivel de endeudamiento merma la capacidad de vivir } \\
\text { saludablemente a la empresa y reduce la velocidad de respuesta a cambios económicos } \\
\text { del entorno. En conclusión, controlar el endeudamiento referido a la capacidad de pago, } \\
\text { mitiga el riesgo de morbilidad frente a cualquier tipo de cambio externo. }\end{array}$ \\
\hline
\end{tabular}




\section{Conclusiones}

El éxito o fracaso de la gestión de una organización está directamente relacionado con la sostenibilidad de la empresa y eventualmente la perdurabilidad de la misma; las empresas que presentan resultados de acuerdo a su planeación estratégica a largo plazo, tienen más posibilidad de perdurar en el tiempo. El reto entonces de los empresarios actuales es constituir y administrar empresas de alto impacto a nivel económico, social y cultural que sean sostenibles y trasciendan más allá de las personas, en otras palabras, empresas perdurables que apliquen e interioricen el concepto de desarrollo sostenible. Finalmente, los empresarios deben propender por generar empresas por oportunidad y no por necesidad, las empresas que se crean como respuesta a la coyuntura social (desempleo) suelen durar menos que las empresas que se crean y constituyen con base en una idea clara de negocio y como respuesta a una oportunidad o a la creación de la oportunidad.

\section{Referencias}

Collins, J., Porras, J. (1997). Built to Last. Estados Unidos: Harper Collins Publishers. Collins, J. (2001). Empresas que sobresalen. Colombia: Editorial Norma.

Da Costa, J. (1992). Readings in mercadeo strategy. Venezuela: Editorial Panapo.

Dávila, C. (1986). El Empresariado colombiano. Una perspectiva histórica. Bogotá: Pontificia Universidad Javeriana.

Delgado, H. (2007). Análisis de estados financieros: finanzas para "no financieros" interpretación para toma de decisiones. México: Editorial Trillas.

Escorche, V. (1990). Productividad y calidad. Argentina: Editorial Nuevos Tiempos.

GIPE. (2005). Factores para la perdurabilidad empresarial. Proyecto de investigación realizado a 318 empresas de Colombia, que pertenecen a los sectores agroindustrial, alimentos, automotor, confecciones, editorial, farmacéutico, financiero, plásticos, servicios, telecomunicaciones y textiles sobre Perdurabilidad. Bogotá: Universidad Del Rosario, Facultad de Administración, Grupo de Investigación en Perdurabilidad.

Jagoe, A. (1987). The winning corporation: management practices that work! Estados Unidos: AcropolisBooks.

Kotler, P. (2010). Caótica: administración y marketing en tiempos de caos. Colombia: Editorial Norma.

Rivera, H. (2009). La perdurabilidad organizacional: un fenómeno explicable desde la biología, la economía y la dirección estratégica de la empresa. Artículo desarrollado como trabajo final del seminario "Teoría organizacional", del Doctorado en Administración de la Universidad de los Andes. Bogotá. 
Rivera, H. (2010). Modelo para que las empresas vivan más tiempo. Universidad, Ciencia y Desarrollo. Programa de Divulgación Científica. Tomo II. Bogotá: Facultad de Administración, Universidad del Rosario.

Scott, E. (2008). Fundamentos de administración financiera. (14a ed). México: Mcgraw-Hill.

Restrepo, L. F., Vélez, R., Méndez, C. E., Rivera, H. A., y Mendoza, L. (2009). Aproximación a una metodología para la identificación de componentes que crean condiciones para la perdurabilidad en empresas colombianas. Documento de investigación (39). Bogotá-Colombia: Editorial Universidad del Rosario.

Cañon L, Vega, C. C. Moreno D (2013). Thanatos empresarial, Muerte empresarial en el sector comercial comportamientos y consecuencias. Bogotá: Facultad de Administración, Universidad del Rosario.

Ramírez G, Muñoz L E y Pulido E (1998). Valorar y comprender una característica fundamental de las organizaciones, su capacidad para permanecer en entornos de diferente complejidad y con grados de turbulencia bastante disímiles. Sotavento 2 31-34 Bogotá

Maturana, H. (2010). La empresa necesita saber qué conservar para sobrevivir (12 de diciembre de 2010) Obtenido de Libre Mercado: http://www.libertaddigital.com/ profesionales/humberto-maturana-la-empresa-necesita-saber-que-conservarpara-sobrevivir-1276409794/ 


\section{Apéndices, tablas, figuras y/o gráficas}

\section{Cuadro 1. Factores para la perdurabilidad empresarial}

\section{COMPONENTE}

1: Identidad organizacional

Siete ítems que incluyen aspectos contemplados en la acción del gobierno de la empresa. Éstos se encuentran incorporados en aspectos éticos, de estrategia, de políticas en la gestión de las personas y del conocimiento del entorno de mercado.
2: Formalización para el gobierno
Cuatro ítems definidos por la formalización de la organización en protocolos de gobierno, su estructura y procesos con un impacto importante en la gestión de las empresas por parte de sus dirigentes.

Cuatro ítems. La cohesión social para la acción, desde la perspectiva de la sociología, señala que el grado de consenso que identifica a un grupo de personas, se manifiesta en una organización en el grado de pertenencia que tienen con sus propósitos y objetivos. Además, indica el nivel de integración que las personas

3: Cohesión social para la acción

4: Formalización, soporte para las decisiones alcanzan en el proceso de interacción social. Los juicios identificados en este componente señalan que la calidad de las relaciones interpersonales, el aprendizaje, la comunicación y el conocimiento, son considerados elementos que contribuyen a la integración de personas, en razón a que por ésta comparten parámetros que guían su acción individual y colectiva como miembros de grupo en la organización.

Tres ítems. Las organizaciones tienen lineamientos establecidos y formalizados que facilitan el proceso de decisiones que en aspectos particulares toman sus directivos en la gestión.

Cuatro juicios o ítems. El reconocimiento en el sector y en el entorno es el resultado de prácticas de gestión que se proyectan en la formalización de sus

5: Reconocimiento por el decisiones y conocimiento, la transparencia en su financiación y el cumplimiento entorno y el sector. del marco legal. Tales prácticas de gestión aplicadas por sus directivos tienen impacto en la sociedad y el sector económico en el que actúa.

Definido por cuatro juicios o ítems. Señalan comportamientos particulares como resultado de políticas para la acción que, por su práctica, permiten diferenciar

6: Diferenciación a la empresa frente a otras con las que compite en el sector al que pertenece. Los juicios de este componente identifican acciones que la empresa tiene que adelantar para identificar oportunidades y ventajas frente a su competencia, con la información que tiene de su mercado, clientes y proveedores.

Dos ítems. El comportamiento social de los empleados en sus procesos de interacción, refleja procesos de carácter asociativo que se proyectan en el proceso

7: Dinámica social de los empleados de cooperación que resulta del trabajo en equipo. Además, los empleados actúan dentro de parámetros que por su empoderamiento propician una dinámica de participación. Esta dinámica social de carácter particular resulta en la organización por la interacción social y por la autonomía.

Cuatro ítems que describen acciones de la empresa referidas al manejo de la información, la incorporación del aprendizaje, la coordinación y la comunicación, así como al comportamiento de socios y directivos en su gobierno. Las situaciones

8: Factores que aportan a la descritas en el Estudio de Condiciones para la Perdurabilidad (ECP) tienen eficiencia relación directa con situaciones particulares que por su presencia contribuyen, en opinión de los expertos consultados con la encuesta, a la eficiencia de los resultados obtenidos.

9: Consolidación

Dos ítems, que enfatiza en la visión de futuro que construyen los directivos como factor que contribuye a su consolidación y la participación de los empleados que crea el compromiso con la empresa. 
10: Gestión Integral

Dos juicios. Se refiere a situaciones que obedecen a políticas de la dirección, como el uso de la planeación estratégica y de la vinculación de los grupos de interés en las decisiones de la empresa. Las circunstancias descritas obedecen a aspectos particulares de la estrategia y el gobierno.

11: Conocimiento de entorno y mercado

12: Eficiencia en procesos

Tres juicios o ítems que hacen referencia, de manera particular, a los comportamientos que los niveles jerárquicos de la organización tienen sobre el mercado y el entorno, así como al impacto que en las ventas tienen las estrategias de inversión en publicidad.

Dos juicios o ítems. Este componente se refiere a que formular procesos dentro de la organización contribuye al ordenamiento de acciones y, que por su impacto, mejoran la eficiencia de la organización.

Identifica que los problemas de liquidez que afectan a la organización se hacen

13: Gestión Financiera evidentes por la aparición de situaciones que la conducen a la mortalidad. En consecuencia, la perdurabilidad de la empresa depende de la gestión financiera y de las estrategias que para esta situación en particular determinen los directivos.

Fuente: Elaboración de los autores propia a partir del Artículo Modelo para que las empresas vivan ,as tiempo. Universidad de Ciencia y Desarrollo, Programa de Divulgación Científica, Universidad del

Rosario, Bogotá, 2008 


\section{Introducción}

Este estudio es resultado de un proceso de investigación desarrollado a partir del proyecto "Vida y muerte empresarial. Estudios empíricos de perdurabilidad" implementado por el Grupo de Investigación en Perdurabilidad Empresarial (GIPE), inscrito en la Línea de Investigación de Realidad Empresarial con el programa Epidemiologia Empresarial de la Escuela de Administración de la Universidad del Rosario. La Escuela se concentró en el reconocimiento a los esfuerzos empresariales de aquellos colombianos que con su ejemplo y resultados han consolidado el crecimiento de sus organizaciones enmarcados en factores de rentabilidad financiera, responsabilidad social y sostenibilidad. Dicha sostenibilidad, usualmente resulta en la creación de riqueza para la nación, en empleo para la población colombiana y de ejemplo para las nuevas generaciones de empresarios. En este contexto, se trataba de comprender el patrón y modelo que ha permitido perdurar a las empresas que ganaron o han estado postuladas al "Premio Empresario Colombiano del Año Mariposa de Lorenz". Este premio ejemplifica la forma como las organizaciones perduran en el tiempo a pesar de las fuerzas externas y su contundencia; cómo se desarrollan, crecen y evolucionan en tiempos de bonanza y en tiempos de dificultad. Reconoce el fortalecimiento de los mecanismos que los empresarios implementan para fomentar una cultura hacia la perdurabilidad empresarial que conduzca a una mayor productividad y competitividad en los sectores económicos del país.

Para ello, se realizó un análisis comparativo entre el modelo de selección del Premio y los trece componentes de medición de condiciones de perdurabilidad y, como resultado, se abordó el esquema y guía mental de la empresa de estudio que le ha dado reconocimiento y permanencia en los mercados. Se incluyó, además, un estudio de dinámica de sistemas para identificar el comportamiento de las empresas ganadoras del premio y el análisis del comportamiento de la empresa a partir de los resultados financieros, con base en los componentes de perdurabilidad. Como resultado, se pretende identificar el patrón y modelo mental que les ha permitido a las empresas permanecer en los mercados. El artículo se enfoca fundamentalmente en los resultados obtenidos para las empresas del Grupo NUTRESA.

\section{Fundamentos teóricos}

Dentro del tema de la problemática empresarial es necesario abordar la búsqueda e identificación de ¿cuáles son los principios administrativos que distinguen a las compañías perdurables de las que no lo son? Este interrogante surge de la observación de los sectores, en los cuales existen ganadores y perdedores, pero ambos se encuentran en un entorno con las mismas características. 
El término de perdurabilidad ha sido abordado desde diferentes autores, entre estos Ramírez, Muñoz y Pulido (1998, p. 10), quienes consideran importante "valorar y comprender una característica fundamental de las organizaciones, su capacidad para permanecer en entornos de diferente complejidad y con grados de turbulencia bastante disímiles". Estos autores buscan una definición que reúna las condiciones que tienen las empresas para perdurar en el tiempo, para identificar características que resultan esenciales para el sostenimiento y éxito de las mismas, y que permita ver cómo a través de los cambios enfrentan problemas que hacen que su gestión sea cada vez más compleja.

El caso de las empresas colombianas ilustra aspectos relacionados con la perdurabilidad y su administración. El Departamento de Planeación Nacional en Colombia (DNP) señala que la pequeña y la mediana empresa difícilmente sobreviven sus primeros años. Según datos recientes, el $41 \%$ no alcanzan al primer año y el $76 \%$ el quinto. La perdurabilidad de las empresas familiares no es mejor. El $35 \%$ sobrevive a la primera generación y el $13 \%$ a la tercera. La búsqueda de respuestas a este hecho conduce a una hipótesis preliminar: las empresas colombianas en su administración, no sólo adolecen de una gestión eficiente de su productividad, sino que además es evidente la ausencia del componente estratégico en el pensamiento de sus dirigentes (Restrepo, 2009).

En el libro Empresas Triunfadoras o en su nombre original The Winning Corporation: Management Practices That Work (Jagoe, 1987), se plantea que la "muerte" de muchas empresas es innecesaria y se puede evitar y, asimismo, presenta una serie de pautas para ayudar a los administradores a planificar el bienestar empresarial en todos los niveles. A su vez, Collins y Porras (1997) publican los hallazgos de una investigación en su libro denominado "Built to Last", seleccionando compañías "visionarias" en lugar de simplemente prósperas o duraderas, para reflejar el hecho de que se han distinguido como un tipo muy especial y selecto de instituciones. Son efectivamente más que prósperas y duraderas, son lo "mejor de lo mejor" en sus respectivas industrias y lo han sido desde hace decenios. Las condiciones establecidas para realizar la investigación fueron: Instituciones líderes en su industria, muy admiradas por personas conocedoras de negocios, que hayan dejado huella indeleble en el mundo en que vivimos, haber tenido múltiples generaciones de presidentes, haber pasado por múltiples ciclos de vida de productos y ser fundadas antes de 1950.

Una década después, Collins (2001) publica "Empresas que Sobresalen" en su nombre en inglés Good to great, en el cuál plasma los resultados de la investigación que buscó identificar las características de las organizaciones que lograron convertir la mediocridad en superioridad. 


\section{Metodología}

El propósito del estudio fue identificar el papel del empresario como guía y estratega en la organización, para lograr no sólo la sostenibilidad de la empresa en el tiempo, sino su éxito en los mercados.

Para efectuarlo, se seleccionó el sector de alimentos - dinámico y de gran impacto socioeconómico en Colombia - tomando como referencia el caso del Grupo Nacional de Chocolates - hoy conocido como GRUPO NUTRESA, el cual ha demostrado durante varias décadas su liderazgo y calidad de vida dentro del sector de alimentos y la perdurabilidad, tanto a nivel nacional como internacional, haciéndolo acreedor de una reputación y ejemplo empresarial para la industria de alimentos. Esta es una de las razones para realizar un estudio acerca de los diferentes factores que hacen que esta compañía se caracterice por la gestión y perdurabilidad en el tiempo. Como apoyo a esta investigación, se realizó una entrevista semiestructurada al Presidente de la organización, junto a una encuesta estructurada a los empleados de cargo profesionales y directivos, en total cuarenta y dos en Bogotá, para así poder analizar las condiciones de perdurabilidad en su interior. Con la entrevista realizada al Presidente del Grupo Nutresa, se vislumbró un panorama amplio sobre las actividades de este grupo y cómo ha logrado ser una de las empresas más exitosas y perdurables del país, que además, ha sido galardonada con el Premio Empresario Colombiano del Año “Mariposa de Lorenz” 2009, entregado por Escuela de Administración de la Universidad del Rosario.

\section{Análisis de datos}

La perdurabilidad es vista como un componente esencial en el desarrollo de las empresas, por esto la Escuela de Administración de la Universidad del Rosario se ha interesado por abordar este tema a fondo, planteando un estudio de "Condiciones para perdurabilidad", que le permitirá entender desde diferentes escenarios el comportamiento de las empresas a través del tiempo. En el marco de las empresas que han estado postuladas al "Premio Empresario del Año Mariposa de Lorenz", se identificaron dos ejes fundamentales que se deben observar: la Dirección y la Gerencia ${ }^{5}$, con el fin de identificar y comprender el patrón y modelo que le ha permitido ser sostenibles en el tiempo. El planteamiento de la Dirección cobra relevancia debido a que los empresarios que se encuentran al frente de su organización deben prospectar el futuro, orientar su consecución y observar la información que

4 Proyecto de investigación, Universidad del Rosario, Escuela de Administración, Grupo de Investigación en Perdurabilidad, realizado a 318 empresas de Colombia, que pertenecen a los sectores agroindustrial, alimentos, automotor, confecciones, editorial, farmacéutico, financiero, plásticos, servicios, telecomunicaciones y textiles. (2005).

5 Información tomada de la página web www.urosario.edu.co 
el entorno le presenta para lograr convertirla en estrategias coherentes y alcanzar un posicionamiento. Entendida así la temática, los trece factores de la perdurabilidad establecidos por la Escuela de Administración se describen en la Tabla 1.

En relación a la Dirección del Grupo Nutresa, en los últimos años se han tomado decisiones que les ha permitido enfrentarse al mundo globalizado, y han sido alineadas de acuerdo a la filosofía de especialización por áreas y objetivos estratégicos de internacionalización para el grupo empresarial, lo que les ha permitido diversificación del riesgo, posicionamiento de marca y distribución en el mercado objetivo. Con respecto a la Gerencia, buscan expandir su mercado, por esto incursiona en el mercado de valores de Colombia como un mecanismo para mejorar su plan de financiamiento y crecimiento. También se enfocan en procesos como: permanente mejora en las relaciones que tiene el grupo con compañías externas como proveedores y clientes, y en procesos internos para mejorar la calidad de los productos, infraestructura, contando con más de doscientos mil puntos de venta donde se distribuyen los productos de las diferentes unidades de negocios. Atienden también con prioridad la gestión humana, siendo muy selectivos a la hora de contratar un nuevo empleado, destacando las cualidades de emprendedores y líderes, seguros de sus opiniones y que puedan aportar a las decisiones de la empresa.

\section{Análisis del sector alimentos en Colombia}

El sector Agroindustrial es importante para el país ${ }^{6}$, debido a que le aporta al PIB nacional el 29\% de la producción total, generando a su vez el $21 \%$ del empleo en Colombia. El sector de alimentos en Colombia se compone de manera porcentual de la siguiente forma: el 29\% corresponde a productos de molinería, el $20 \%$ a productos lácteos, el $18 \%$ a carnes y pescados, el $15 \%$ a aceites, hortalizas, flores y grasas, el $8 \%$ a productos de panadería y el 10\% representa a otros subsectores. En 2010, el sector de alimentos creció a una tasa anual del 2,3\% y el de bebidas se contrajo a una tasa del 2,5\%. Ambos sectores crecieron en este período por debajo del promedio de la industria que creció a una tasa del 4,5\%. Durante el primer semestre de 2011, el crecimiento del sector de alimentos fue de 3,1\%, levemente inferior al promedio industrial que fue del 3,5\% especialmente por los sectores de molinería y almidones, refinación de azúcar, y transformación y conservación de carne y pescado.

El crecimiento del sector de alimentos desde el 2011 ha sido impulsado por la reactivación de la demanda interna, y por el dinamismo de las exportaciones que han sido muy dinámicas desde ese período. El crecimiento del sector ha sido frenado en los últimos años por las emergencias invernales que afectan especialmente a sectores como el de productos cárnicos, refinación de azúcar, productos lácteos y bebidas. Al ubicar el sector de molinería, panadería y almidones, se puede afirmar que ha

6 Ver Sector Agroindustrial Colombiano. http://www.inviertaencolombia.com.co 
presentado un crecimiento sobrio debido al costo de la materia prima proveniente del exterior, entre las que se encuentran el trigo, principal insumo para la producción, el maíz y la soya. Pero dentro de este sector sobresale de manera favorable el subsector de galletería, debido al importante volumen de exportaciones. Asimismo, se encuentra el subsector de cacao y chocolatería que ha presentado durante los últimos años una actividad creciente, en gran medida debido a su innovación, comercialización, diversificación y diferenciación de productos en el territorio nacional y su expansión a otros países. Aunque este sector ha sido negativamente afectado por la volatilidad y aumento en los precios del azúcar y la leche, los precios poco competitivos del cacao y las limitaciones en materia de producción, así como la implementación de mejores tecnologías, ha logrado sostenerse dentro de los más importantes y que aportan de manera significativa al PIB del país.

\section{Historia del Grupo Nutresa}

Desde el punto de vista histórico, este grupo antiqueño fue fundado en 1916 por varios empresarios inicialmente como productora de galletas y confetis bajo el nombre de "Fábrica Nacional de Galletas y Confites". En 1933 se hizo accionista por medio de la adquisición de nueva tecnología y fabricación de la marca Dux de Galletas Noel. En 1958, como parte de su estrategia de Grupo se crearon las Marcas Sello Rojo y Colcafé, con el fin de comercializar café tostado, soluble y molido, generando así más ingresos a la compañía. En 1960 se fusionaron dos filiales dándole como origen a la Industria Colombia de Café S.A, ese mismo año adquirieron Alimentos Zenú y Alimentos Cárnicos. En 1968 realizaron una adquisición nacional con la compra de la Fábrica Café de la Bastilla, la cual se transformó en Tropical Coffee Company. En 1970 aumentaron las adquisiciones a nivel nacional, identificando la primera en Barranquilla donde compró Frigorífico Continental y la segunda en Bogotá a través de Frigorífico Suizo con el fin de mejorar la oferta de productos cárnicos. En 1980 adquirió y fundó dos empresas, de galletas y cárnicos, Molinos de Santa Marta como proveedor de harina de trigo y Tecniagro. Con la apertura económica de los años 90, comenzaron a incursionar en el mercado internacional fundando comercializadoras en Venezuela y Ecuador. En 1997 adquirieron los Productos Alimenticios Doria, empresa líder en el mercado de las pastas; en 1999 realizó un Joint Venture vendiéndole a Danone el 30\% de Galletas Noel con el fin de generar un aliado estratégico.

A principios del nuevo milenio se dio paso a la venta de los productos por medio de “canales alternativos", caracterizados por máquinas dispensadoras y por catálogos. El año 2002 fundó una nueva comercializadora en México, la cual le abría paso para llegar a este país así como varias escisiones con el fin de mejorar la participación en los mercados, y en el 2004 las comercializadoras Cordialsa en Estados Unidos, Panamá, 
Guatemala, Costa Rica, Nicaragua, Honduras y El Salvador. Se compraron las fábricas de galletas y chocolates de Nestlé en Costa Rica, posicionando a la Compañía Grupo Nacional de Chocolates y Grupo Nacional de Galletas en este mercado. En 2005 la casa matriz se hizo dueña del $100 \%$ de las filiales adquiriendo de nuevo el $30 \%$ de Galletas Noel, y se fundaron dos nuevas filiales, como es el caso de Pastas Commarico y Setas de Colombia; y a nivel internacional se adquirieron Galletas Pozuelo en Costa Rica y Blue Ribbon en Panamá. En 2007 con ansias de mejorar la eficiencia y efectividad comercial, se fusionaron las redes de distribución de Nicaragua y Panamá. En 2008 se generó un Joint Venture con De La Recetta y Alpina para darle mayor cubrimiento al mercado colombiano. En 2009 se creó el Grupo Nutresa de México, y en el 2010 adquirió galletas dulces llamada Ferh Holdings LLC. Finalmente, en el 2011 se adquirió en República Dominicana la compañía especializada en helados llamada Helados Bon. En este mismo año, el Grupo Nacional de Chocolates dejó de llamarse así para cambiar a Grupo Nutresa, debido a que su propósito era una imagen y razón social que reflejara todas sus unidades de negocio y el compromiso que tiene con la nutrición.

\section{Resultados}

¿Por qué el Grupo Nutresa es una empresa perdurable? De acuerdo al contexto de la organización, el Grupo Nutresa es una empresa con objetivos empresariales orientados a la competitividad y por ende, a la perdurabilidad. Los objetivos estratégicos están enfocados a seguir creciendo exitosamente, fortaleciendo factores como competitividad, salud financiera, cultura empresarial, capacidad de innovación, capacidad de transformación y de mirar al futuro. La adquisición de talento humano joven y renovado; las marcas de la empresa que han cambiado de acuerdo a la época y necesidades del consumidor; la existencia de un solo código de Gobierno corporativo continuo donde la empresa se constituye como la única sociedad inversionista y se incrementa la eficiencia y la simplicidad; la capacidad de la compañía de transformarse y adaptarse a los tiempos, el que Grupo Nutresa sea una compañía con una capacidad de distribución que abarca el territorio nacional colombiano y todos los países donde se ha expandido y por último, la innovación de sus productos, procesos y modelo de negocios que la diferencian en el mercado, son factores definitivos para que clasifique dentro de las empresas perdurables.

Como resultado, el Grupo Nutresa expone sus teorías frente a los 13 ítems de perdurabilidad: 


\begin{tabular}{|c|c|}
\hline $\begin{array}{c}1 . \\
\text { Identidad de la } \\
\text { organización: }\end{array}$ & $\begin{array}{l}\text { La identidad organizacional en particular son los valores, comportamientos éticos y la } \\
\text { responsabilidad social identificados al interior de la organización. Cabe resaltar que } \\
\text { los valores que componen la filosofía y la actuación corporativa son: la autonomía y } \\
\text { coherencia estratégica, el buen gobierno corporativo, la competitividad, el desarrollo } \\
\text { de los trabajadores, el desarrollo sostenible, la ética, la gestión de la innovación y } \\
\text { el conocimiento, la participación, agilidad y gestión colaborativa, el respeto y la } \\
\text { seguridad alimentaria. Con estos componentes de cultura organizacional se encuentran } \\
\text { comprometidos los } 28.500 \text { trabajadores del Grupo Nutresa, lo que hace que la } \\
\text { organización esté orientada a un mismo propósito, con políticas de gestión alineada } \\
\text { con los resultados extraordinarios y a la adaptabilidad frente al cambio del entorno del } \\
\text { mercado, factores claves para llegar al camino de la perdurabilidad. }\end{array}$ \\
\hline $\begin{array}{c}2 . \\
\text { Formalización } \\
\text { para el gobierno }\end{array}$ & $\begin{array}{l}\text { El Grupo Nutresa se ha caracterizado por una longevidad de } 90 \text { años; para las empresas } \\
\text { líderes del mundo el promedio es de } 40 \text { años, por lo cual este indicador refleja la buena } \\
\text { salud empresarial de la compañía. Durante los } 90 \text { años de vida de la compañía, el } \\
\text { gobierno corporativo ha sido continuo y no generacional. Al ser continuo, significa que } \\
\text { los principios de la identidad organizacional han evolucionado con el tiempo de acuerdo } \\
\text { a la demanda del mercado y a los marcos regulatorios. }\end{array}$ \\
\hline $\begin{array}{c}3 . \\
\text { Cohesión social } \\
\text { para la acción }\end{array}$ & $\begin{array}{l}\text { La cohesión social se vivencia en la organización a través de los incentivos que se otorgan } \\
\text { al alcanzar resultados. Dentro de estos incentivos se encuentran las bonificaciones, que } \\
\text { dependen efectivamente del éxito en ventas. La medición del clima organizacional } \\
\text { muestra que la organización se encuentra en un nivel sobresaliente. Esto comprueba } \\
\text { que la fuerza de la cultura organizacional a través de una evaluación sobre la claridad } \\
\text { en los objetivos, las condiciones de trabajo, el apoyo del jefe directo, la remuneración, el } \\
\text { reconocimiento, la formación y la capacitación así como talento motivado y formado, se } \\
\text { encamina a obtener los estándares más altos. }\end{array}$ \\
\hline $\begin{array}{l}4 . \\
\text { Formalización: } \\
\text { soporte para las } \\
\text { decisiones: }\end{array}$ & $\begin{array}{l}\text { A lo largo de su vida organizacional, el grupo se ha caracterizado por la especialización } \\
\text { en diferentes áreas que le han permitido responder rápidamente a cambios en el entorno } \\
\text { bajo un enfoque de gobierno corporativo continuo. Bajo la creación en el } 2006 \text { de } \\
\text { Servicios Nacional de Chocolates S.A, el centro de servicios compartidos del Grupo y } \\
\text { con la fusión en el } 2007 \text { de las redes de distribución Cordialsa y Pozuelo en Nicaragua } \\
\text { y Panamá respectivamente, ha logrado mayor eficiencia y efectividad comercial para la } \\
\text { organización. Por otro lado, los lineamientos establecidos para la toma de decisiones } \\
\text { del grupo, han propiciado la supervivencia de la compañía en Venezuela, gracias } \\
\text { a la ideología políticamente neutral y el mantenimiento de precios inferiores a los de } \\
\text { la competencia, lo que ha mitigado los posibles riesgos de expropiación por parte del } \\
\text { gobierno de este país. }\end{array}$ \\
\hline $\begin{array}{c}5 . \\
\text { Reconocimiento } \\
\text { por el entorno y el } \\
\text { Sector: }\end{array}$ & $\begin{array}{l}\text { Este ítem de perdurabilidad se encuentra íntimamente relacionado con el objetivo } \\
\text { estratégico de crecimiento y liderazgo en el mercado por parte del grupo; objetivo } \\
\text { orientado a incrementar el tamaño y la participación del mismo. Para lograr este objetivo } \\
\text { se requiere ser líderes, hoy el Grupo Nutresa se constituye como un seguidor de esta } \\
\text { filosofía, sumado al objetivo estratégico de internacionalización, el cual está orientado } \\
\text { a la diversificación y a la disminución de la concentración en un solo mercado, con el } \\
\text { fin de reducir vulnerabilidades de la empresa y, por ende, al incremento del tamaño del } \\
\text { mercado objetivo. }\end{array}$ \\
\hline $\begin{array}{c}6 . \\
\text { Diferenciación }\end{array}$ & $\begin{array}{l}\text { En los últimos años se han realizado catorce adquisiciones de compañías nacionales } \\
\text { e internacionales por parte del grupo, lo que refleja innovación, nuevas marcas y un } \\
\text { portafolio de productos mucho más fuerte y sólido en un mercado cada vez más incierto. } \\
\text { Se destaca que esta diferenciación se fundamenta en la creación de valor agregado y } \\
\text { no en el sostenimiento de bajos costos, lo que garantiza que el Grupo Nutresa no se vea } \\
\text { afectada por los efectos de la revaluación y por los altos costos que significa la existencia } \\
\text { de la protección efectiva negativa para el sector agrícola y, por tanto, posea un margen } \\
\text { de rentabilidad para soportar los fenómenos externos y sobrevivir. }\end{array}$ \\
\hline
\end{tabular}




\begin{tabular}{|c|c|}
\hline $\begin{array}{l}7 . \\
\text { Dinámica social de } \\
\text { los empleados }\end{array}$ & $\begin{array}{l}\text { Uno de los objetivos estratégicos del Grupo Nutresa está orientado al desarrollo de una } \\
\text { filosofía de gestión humana enfocada en la vinculación de la persona y el camino del } \\
\text { conocimiento dentro del factor humano, con el propósito de atraer y retener el talento } \\
\text { dentro de la organización. } \\
\text { La empresa propicia condiciones para la eficiencia y calidad en la acción de sus empleados } \\
\text { mediante la participación activa y el empoderamiento que les da en las decisiones, así } \\
\text { como la integración de grupos de trabajo, la consolidación de la cooperación y del } \\
\text { trabajo en equipo. De esta manera, resulta una dinámica social producto de la interacción } \\
\text { social y la autonomía. } \\
\text { El empoderamiento en los empleados que comparten un proceso de trabajo cualquiera, } \\
\text { hace que estos asuman la responsabilidad por la marcha de la actividad, tomando todas } \\
\text { las medidas que resulten necesarias, siempre que estén alineadas con la misión de su } \\
\text { unidad, la que a su vez debe ser congruente con la misión general de la empresa. } \\
\text { Al generar las condiciones propicias para la retención de talento humano, es claro que } \\
\text { las raíces de la cultura organizacional van a estar más arraigadas en una organización } \\
\text { que propicie el bienestar de sus empleados, lo que conlleva a trabajar con mayor } \\
\text { perseverancia por los objetivos comunes y la misión y visión de la organización. }\end{array}$ \\
\hline $\begin{array}{l}8 . \\
\text { Factores que } \\
\text { aportan a la } \\
\text { eficiencia }\end{array}$ & $\begin{array}{l}\text { El paso del gobierno corporativo de sociedades anónimas a holdings, donde el Grupo } \\
\text { Nacional Chocolates es el dueño único del } 100 \% \text { de las filiales, permitió la reducción } \\
\text { de juntas directivas innecesarias, creando juntas nominales y reducción de los auditores } \\
\text { externos, dejando un sólo inversionista llamado GNC (Grupo Nacional de Chocolates). }\end{array}$ \\
\hline $\begin{array}{c}9 . \\
\text { Consolidación }\end{array}$ & $\begin{array}{l}\text { La consolidación del grupo es resultado de la construcción de una organización con una } \\
\text { visión de futuro que hacen sus directivos, así como del compromiso y participación de } \\
\text { los empleados en ella. Es así como el objetivo para el } 2015 \text { es proporcionar calidad de } \\
\text { vida al consumidor con productos que satisfagan sus aspiraciones de bienestar, nutrición } \\
\text { y placer, lo que refleja crecimiento, consolidación y perdurabilidad. }\end{array}$ \\
\hline $\begin{array}{c}10 . \\
\text { Gestión Integral }\end{array}$ & $\begin{array}{l}\text { Dentro de los objetivos estratégicos del Grupo Nutresa se encuentra el desarrollo } \\
\text { sostenible que comprende responsabilidad social y ambiental. En este sentido, el grupo en } \\
\text { el } 2006 \text { creó la Fundación Grupo Nacional de Chocolates, con el propósito de concentrar } \\
\text { los esfuerzos de las compañías del Grupo en su contribución al desarrollo económico y } \\
\text { social de la comunidad. Adicionalmente creó el Centro de Investigación en Nutrición, } \\
\text { Salud y Bienestar Vidarium, que posteriormente se transforma en la Corporación que } \\
\text { lleva su mismo nombre en el } 2008 \text {. }\end{array}$ \\
\hline $\begin{array}{l}\text { Conocimiento } \\
\text { del entorno y el } \\
\text { mercado }\end{array}$ & $\begin{array}{l}\text { Bajo cada línea de negocio, el Grupo Nutresa cuenta con equipos e investigación y } \\
\text { desarrollo que continuamente intentan mejorar los productos y los ajustan a las nuevas } \\
\text { tendencias del mercado. }\end{array}$ \\
\hline $\begin{array}{l}12 . \\
\text { Eficiencia en } \\
\text { procesos }\end{array}$ & $\begin{array}{l}\text { Los métodos de gestión se materializan con la restructuración del gobierno corporativo, } \\
\text { en donde día a día, con el crecimiento y adquisiciones que el Grupo Nutresa realiza } \\
\text { a lo largo de Latinoamérica, se genera un enfoque especializado donde prima el } \\
\text { empoderamiento de los empleados en todos los niveles, llevando a que exista eficiencia } \\
\text { al interior de la organización. }\end{array}$ \\
\hline $\begin{array}{l}13 . \\
\text { Gestión } \\
\text { Financiera }\end{array}$ & $\begin{array}{l}\text { Uno de los objetivos estratégicos de la organización es el crecimiento rentable. Este } \\
\text { objetivo permite que el consumidor adquiera los productos, buscando no elevar tanto } \\
\text { el nivel de apalancamiento conservador e inferior al } 2 \% \text { para el grupo. Esto debido a } \\
\text { que para la organización un alto nivel de endeudamiento merma la capacidad de vivir } \\
\text { saludablemente a la empresa y reduce la velocidad de respuesta a cambios económicos } \\
\text { del entorno. En conclusión, controlar el endeudamiento referido a la capacidad de pago, } \\
\text { mitiga el riesgo de morbilidad frente a cualquier tipo de cambio externo. }\end{array}$ \\
\hline
\end{tabular}




\section{Conclusiones}

El éxito o fracaso de la gestión de una organización está directamente relacionado con la sostenibilidad de la empresa y eventualmente la perdurabilidad de la misma; las empresas que presentan resultados de acuerdo a su planeación estratégica a largo plazo, tienen más posibilidad de perdurar en el tiempo. El reto entonces de los empresarios actuales es constituir y administrar empresas de alto impacto a nivel económico, social y cultural que sean sostenibles y trasciendan más allá de las personas, en otras palabras, empresas perdurables que apliquen e interioricen el concepto de desarrollo sostenible. Finalmente, los empresarios deben propender por generar empresas por oportunidad y no por necesidad, las empresas que se crean como respuesta a la coyuntura social (desempleo) suelen durar menos que las empresas que se crean y constituyen con base en una idea clara de negocio y como respuesta a una oportunidad o a la creación de la oportunidad.

\section{Referencias}

Collins, J., Porras, J. (1997). Built to Last. Estados Unidos: Harper Collins Publishers. Collins, J. (2001). Empresas que sobresalen. Colombia: Editorial Norma.

Da Costa, J. (1992). Readings in mercadeo strategy. Venezuela: Editorial Panapo.

Dávila, C. (1986). El Empresariado colombiano. Una perspectiva histórica. Bogotá: Pontificia Universidad Javeriana.

Delgado, H. (2007). Análisis de estados financieros: finanzas para "no financieros" interpretación para toma de decisiones. México: Editorial Trillas.

Escorche, V. (1990). Productividad y calidad. Argentina: Editorial Nuevos Tiempos.

GIPE. (2005). Factores para la perdurabilidad empresarial. Proyecto de investigación realizado a 318 empresas de Colombia, que pertenecen a los sectores agroindustrial, alimentos, automotor, confecciones, editorial, farmacéutico, financiero, plásticos, servicios, telecomunicaciones y textiles sobre Perdurabilidad. Bogotá: Universidad Del Rosario, Facultad de Administración, Grupo de Investigación en Perdurabilidad.

Jagoe, A. (1987). The winning corporation: management practices that work! Estados Unidos: AcropolisBooks.

Kotler, P. (2010). Caótica: administración y marketing en tiempos de caos. Colombia: Editorial Norma.

Rivera, H. (2009). La perdurabilidad organizacional: un fenómeno explicable desde la biología, la economía y la dirección estratégica de la empresa. Artículo desarrollado como trabajo final del seminario "Teoría organizacional", del Doctorado en Administración de la Universidad de los Andes. Bogotá. 
Rivera, H. (2010). Modelo para que las empresas vivan más tiempo. Universidad, Ciencia y Desarrollo. Programa de Divulgación Científica. Tomo II. Bogotá: Facultad de Administración, Universidad del Rosario.

Scott, E. (2008). Fundamentos de administración financiera. (14a ed). México: Mcgraw-Hill.

Restrepo, L. F., Vélez, R., Méndez, C. E., Rivera, H. A., y Mendoza, L. (2009). Aproximación a una metodología para la identificación de componentes que crean condiciones para la perdurabilidad en empresas colombianas. Documento de investigación (39). Bogotá-Colombia: Editorial Universidad del Rosario.

Cañon L, Vega, C. C. Moreno D (2013). Thanatos empresarial, Muerte empresarial en el sector comercial comportamientos y consecuencias. Bogotá: Facultad de Administración, Universidad del Rosario.

Ramírez G, Muñoz L E y Pulido E (1998). Valorar y comprender una característica fundamental de las organizaciones, su capacidad para permanecer en entornos de diferente complejidad y con grados de turbulencia bastante disímiles. Sotavento 2 31-34 Bogotá

Maturana, H. (2010). La empresa necesita saber qué conservar para sobrevivir (12 de diciembre de 2010) Obtenido de Libre Mercado: http://www.libertaddigital.com/ profesionales/humberto-maturana-la-empresa-necesita-saber-que-conservarpara-sobrevivir-1276409794/ 


\section{Apéndices, tablas, figuras y/o gráficas}

\section{Cuadro 1. Factores para la perdurabilidad empresarial}

COMPONENTE

1: Identidad organizacional

Siete ítems que incluyen aspectos contemplados en la acción del gobierno de la empresa. Éstos se encuentran incorporados en aspectos éticos, de estrategia, de políticas en la gestión de las personas y del conocimiento del entorno de mercado.

2: Formalización para el gobierno

Cuatro ítems definidos por la formalización de la organización en protocolos de gobierno, su estructura y procesos con un impacto importante en la gestión de las empresas por parte de sus dirigentes.
3: Cohesión social para la acción

4: Formalización, soporte para las decisiones

5: Reconocimiento por el entorno y el sector.

6: Diferenciación

7: Dinámica social de los empleados

8: Factores que aportan a la eficiencia

9: Consolidación
Cuatro ítems. La cohesión social para la acción, desde la perspectiva de la sociología, señala que el grado de consenso que identifica a un grupo de personas, se manifiesta en una organización en el grado de pertenencia que tienen con sus propósitos y objetivos. Además, indica el nivel de integración que las personas alcanzan en el proceso de interacción social. Los juicios identificados en este componente señalan que la calidad de las relaciones interpersonales, el aprendizaje, la comunicación y el conocimiento, son considerados elementos que contribuyen a la integración de personas, en razón a que por ésta comparten parámetros que guían su acción individual y colectiva como miembros de grupo en la organización.

Tres ítems. Las organizaciones tienen lineamientos establecidos y formalizados que facilitan el proceso de decisiones que en aspectos particulares toman sus directivos en la gestión.

Cuatro juicios o ítems. El reconocimiento en el sector y en el entorno es el resultado de prácticas de gestión que se proyectan en la formalización de sus decisiones y conocimiento, la transparencia en su financiación y el cumplimiento del marco legal. Tales prácticas de gestión aplicadas por sus directivos tienen impacto en la sociedad y el sector económico en el que actúa.

Definido por cuatro juicios o ítems. Señalan comportamientos particulares como resultado de políticas para la acción que, por su práctica, permiten diferenciar a la empresa frente a otras con las que compite en el sector al que pertenece. Los juicios de este componente identifican acciones que la empresa tiene que adelantar para identificar oportunidades y ventajas frente a su competencia, con la información que tiene de su mercado, clientes y proveedores.

Dos ítems. El comportamiento social de los empleados en sus procesos de interacción, refleja procesos de carácter asociativo que se proyectan en el proceso de cooperación que resulta del trabajo en equipo. Además, los empleados actúan dentro de parámetros que por su empoderamiento propician una dinámica de participación. Esta dinámica social de carácter particular resulta en la organización por la interacción social y por la autonomía.

Cuatro ítems que describen acciones de la empresa referidas al manejo de la información, la incorporación del aprendizaje, la coordinación y la comunicación, así como al comportamiento de socios y directivos en su gobierno. Las situaciones descritas en el Estudio de Condiciones para la Perdurabilidad (ECP) tienen relación directa con situaciones particulares que por su presencia contribuyen, en opinión de los expertos consultados con la encuesta, a la eficiencia de los resultados obtenidos.

Dos ítems, que enfatiza en la visión de futuro que construyen los directivos como factor que contribuye a su consolidación y la participación de los empleados que crea el compromiso con la empresa. 
Dos juicios. Se refiere a situaciones que obedecen a políticas de la dirección, como el uso de la planeación estratégica y de la vinculación de los grupos de interés en las decisiones de la empresa. Las circunstancias descritas obedecen a aspectos particulares de la estrategia y el gobierno.

11: Conocimiento de entorno y mercado

12: Eficiencia en procesos

Tres juicios o ítems que hacen referencia, de manera particular, a los comportamientos que los niveles jerárquicos de la organización tienen sobre el mercado y el entorno, así como al impacto que en las ventas tienen las estrategias de inversión en publicidad.

Dos juicios o ítems. Este componente se refiere a que formular procesos dentro de la organización contribuye al ordenamiento de acciones y, que por su impacto, mejoran la eficiencia de la organización.

Identifica que los problemas de liquidez que afectan a la organización se hacen

13: Gestión Financiera evidentes por la aparición de situaciones que la conducen a la mortalidad. En consecuencia, la perdurabilidad de la empresa depende de la gestión financiera y de las estrategias que para esta situación en particular determinen los directivos.

Fuente: Elaboración de los autores propia a partir del Artículo Modelo para que las empresas vivan ,as tiempo. Universidad de Ciencia y Desarrollo, Programa de Divulgación Científica, Universidad del

Rosario, Bogotá, 2008 